\title{
INTEGRO-DIFFERENTIAL CHAPMAN-KOLMOGOROV EQUATION FOR CONTINUOUS-JUMP MARKOV PROCESSES AND ITS USE IN PROBLEMS OF MULTI-COMPONENT RENEWAL IMPULSE PROCESS EXCITATIONS
}

\author{
R. Iwankiewicz \\ Hamburg University of Technology, Hamburg, Germany
}

\section{Introduction}

State vector of a dynamic system under a Poisson train of impulses is a non-diffusive Markov process and its joint probability density function satisfies an integro-differential generalized

Fokker-Planck-Kolmogorov equation which is also called Kolmogorov-Feller equation (cf. e.g. [1]). If the train of impulses is driven by non-Poisson, for example renewal, counting processes, the state vector is not a Markov process. For some classes of non-Poisson counting processes the nonMarkov pulse problems can be converted into the Markov ones by augmenting the state vector of the dynamic system by auxiliary variables driven by either a single or two independent Poisson processes. Exact techniques of this kind have been developed for trains of impulses driven by Erlang renewal processes [2,3] or by a generalized Erlang renewal process [4]. As the augmented state vector is Poisson-driven, the differential equations for response moments can be derived. In all those impulse process excitations problems the displacement response is continuous, the velocity response is continuous-jump and the auxiliary variables are purely jump processes. As those auxiliary jump processes are driven by Poisson processes, they can be alternatively characterized in terms of a number of Markov states. Hence, the problem is jointly described by the original state variables and the Markov states of the auxiliary jump process. Accordingly, the response probability distribution may be characterized by a joint probability density-distribution of the response variables and of the states of a pertinent Markov chain. The fundamental equation for continuous-jump Markov processes is the general integro-differential Chapman-Kolmogorov equation [5]. The explicit integro-differential equations governing the joint probability densitydistribution of the response are obtained from the Chapman-Kolmogorov equations, after the determination of the jump probability intensity functions for the continuous-jump and purely jump processes. The explicit equations governing the response probability density have been derived for oscillators under random trains of impulses driven by single, renewal processes $[6,7]$.

\section{Statement of the problem}

In the present paper the approach to the excitation impulse process based on the integrodifferential Chapman-Kolmogorov equation is extended from a single renewal impulse process to a a multi-component one. First, the integro-differential Chapman-Kolmogorov equation is introduced and its use for purely jump processes is demonstrated. The examples of purely jump stochastic processes are the Poisson counting process and a two-state Markov process (a rectangular wave process). The considered impulse excitation consists of a number of $\mathbf{n}$ random trains of impulses, each of whom is driven by an Erlang renewal process with parameter k(i). The driving processes are assumed to be statistically independent. Each of the impulse processes is recast into a Poisson driven impulse process, with the aid of auxiliary, purely jump stochastic variables, each of whom is governed by a stochastic differential equation driven by the $\mathrm{i}$-th Poisson process. 
As each i-th Erlang impulse process is characterized by k(i) "phases", a chain of k(i) Markov states is associated with it. The Markov chain for the whole problem is constructed by considering the coincidences of the states of the individual jump processes. Thus the total number of Markov states is determined. The jump probability intensity functions pertinent to this problem are formulated. The explicit equations governing the joint probability density-distribution function of the response and of the Markov states of the auxiliary jump variables are derived from the general integro-differential forward Chapman-Kolmogorov equation via the integrations over the state space. The resulting equations form a set of integro-partial differential equations.

\section{References}

[1] A. Renger (1979). Equation for probability density of vibratory systems subjected to continuous and discrete stochastic excitation, Zeitschrift fuer Angewandte Mathematik und Mechanik, 59, 1-13,

[2] S.R.K. Nielsen, R. Iwankiewicz and P.S. Skjaerbaek (1995). Moment equations for non-linear systems under renewal-driven random impulses with gamma-distributed interarrival times. In: Proc. of IUTAM Symposium on Advances in Nonlinear Mechanics, Trondheim, Norway, Eds.A. Naess and S. Krenk, Kluwer, 331-340.

[3] R. Iwankiewicz and S.R.K. Nielsen (1999). Advanced methods in stochastic dynamics of non-linear systems, Aalborg University Press.

[4] R. Iwankiewicz (2002). Dynamic systems under random impulses driven by a generalized Erlang renewal process, Proc. of 10th IFIP WG 7.5 Working Conference on Reliability and Optimization of Structural Systems, Osaka, Japan. Eds. H.Furuta, M.Dogaki and M.Sakano, Balkema, 103-110.

[5] C.W. Gardiner (1985). Handbook of stochastic methods for physics, chemistry and the natural sciences, Springer-Verlag.

[6] R. Iwankiewicz (2005). Equation for probability density of the response of a dynamic system to Erlang renewal random impulse processes. Proc. of 12th IFIP WG 7.5 Working Conference on Reliability and Optimization of Structural Systems, Aalborg, Denmark, Eds. J.D. Soerensen and D.M. Frangopol, Taylor \& Francis, 107-113.

[7] R. Iwankiewicz (2008). Equations for probability density of response of dynamic systems to a class of non-Poisson random impulse process excitations, Probabilistic Engineering Mechanics, 23 ( 2-3), 198-207. 